\title{
Altın Çakal'da (Canis aureus Linnaeus, 1758) Bulunan Helmint Enfeksiyonları ve Zoonotik Önemi
}

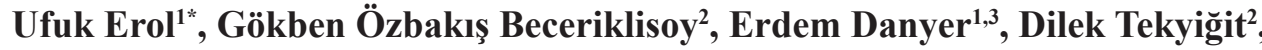 \\ H. Oğuz Sarımehmetoğlu² \\ ${ }^{1}$ Veteriner Kontrol Merkez Araştırma Enstitüsü, Parazitoloji Laboratuvarl, Ankara. \\ ${ }^{2}$ Ankara Üniversitesi Veteriner Fakültesi, Parazitoloji Anabilim Dall, Ankara. \\ ${ }^{3}$ Türk Deniz Araştırmaları Vakfi, İstanbul.
}

Geliş Tarihi / Received: 18.04.2019, Kabul Tarihi / Accepted: 14.05.2019

\begin{abstract}
Özet: Altın çakal (Canis aureus Linnaeus, 1758), Türkiye'de bulunan, dünyada da oldukça geniş yayılıma sahip yabani karnivorlardan biridir. Yabani karnivorlar çeşitli zoonoz helmintleri çevreye, insanlara ve diğer hayvanlara bulaştırmaları bakımından önemli bir kaynak oluşturmaktadır. Bu araştırmada, çakallarda bulunabilecek, çevreye bulaşarak insan ve hayvan sağlığını tehdit edecek helmintlerin varlığı aranmaktadır. Bu amaçla, Türkiye'nin Batı Karadeniz ve Güney Marmara bölgesinden Veteriner Kontrol Merkez Araştırma Enstitüsü’ne gönderilen iki çakal incelenmiş, sindirim sistemlerinde; Alaria sp., Joyeuxiella echinorhyncoides, Mesocestoides sp., Uncinaria stenocephala, Rictularia cahirensis erişkinleri ile, Linguatula serrata nimfleri saptanmıştır. Bu çalışmada tespit edilen Alaria sp. ve L. serrata nimfleri bildiğimiz kadarıyla Türkiye'de altın çakallardan bildirilen ilk kayıttır.
\end{abstract}

Anahtar kelimeler: Altın çakal, Helmint, Linguatula serrata, Alaria sp., Zoonoz Helmintler

\section{Helminth Infections in Golden Jackals (Canis aureus Linnaeus, 1758) and Their Zoonotic Importance}

\begin{abstract}
Golden jackal (Canis aureus Linnaeus,1758) is one of the wild carnivore in the worldwide spread. Wild carnivores are an important source of transmission of various zoonotic helminths to the environment, humans and other animals. In this study, it was aimed to diagnose helminths which can be found in golden jackals and will threaten human and animal health by transmitting to the environment. For this purpose, two jackals which were brought from Western Blacksea and Southern Marmara region of Turkey to the Veterinary Control Central Research Institute were examined; and adults of Alaria sp., Joyeuxiella echinorhyncoides, Mesocestoides sp., Uncinaria stenocephala, Rictularia cahirensis and Linguatula serrata nymphs had been found in their gastrointestinal system. To the best of our knowledge, it is the first record of Alaria sp. and L. serrata nymphs in golden jackals from Turkey.
\end{abstract}

Key words: Golden Jackals, Helminth, Linguatula serrata, Alaria sp., Zoonotic helminths

\section{Giriş}

Altın çakal, mesokarnivor türlerinden olup, dünyada geniş yayılıma sahip karnivor türlerindedir [14]. Polytypic tür olan altın çakalın, 14 alt türü farklı coğrafik bölgelerde bulunmaktadır [10]. Altın çakal Afrika, Güney Doğu Asya, Orta Asya, Orta Doğu, Basra Körfezi ülkelerinde [14] ve Kuzey İtalya, Slovakya, Avusturya, Macaristan, Çek Cumhuriyeti, Kuzey Polonya, Slovenya, Romanya, Bosna-Hersek, Karadağ, Sirbistan, Ukrayna, Yunanistan, Bulgaristan ve Türkiye'de yaşadığı bilinmektedir [4].

Türkiye'de oldukça geniş bir alanda yaşam sürdüren altın çakallar özellikle sahil bölgelerinde ve deniz seviyesinden 1500 metre yükseklikteki nehir vadilerinde (Kocaeli, İstanbul, Kırklareli, Sakarya, Ankara, Adana, Mersin, Hatay, Gaziantep, Şanlıurfa, İzmir, Denizli, Muğla, Aydın, Burdur, Zonguldak, Karabük, Bolu, Artvin, Rize, Trabzon, Giresun, Ordu, Samsun, Kastamonu, Bartın ve küçük bir popülasyon halinde Hakkari'de) bulunmaktadır [2].

Altın çakallar, $1,1 \mathrm{~km}^{2}$ 'den $20 \mathrm{~km}^{2}$ 'ye uzanan bir yaşam alanına sahiptir. Bu durum çakalların çeşitli parazitler türleri dışında diğer karnivorlar ile de temas ederek taşıdığ etkenlerini daha geniş alanlara yayılma ihtimalini arttırmaktadır. Parazitlerin çevreye bulaşmasında ve insanlar için indirekt enfeksiyon kaynağı olmasında önemli rolü olduğu düşünülmektedir [10]. Altın ça- 
kallarda yapılan parazitolojik çalışmalar çoğunlukla helmintler üzerine olup, 38 ülkeden 119 helmint türü bildirilmiş ve bu helmint türleri içerisinde zoonoz öneme sahip olanlar da kaydedilmiştir [10].

Türkiye'nin Avrupa'daki komşusu Bulgaristan, Yunanistan ve doğu sınır komşusu İran'da çakal parazitlerinin yayıllışı ile ilgili çeşitli çalışmalar bulunmasına rağmen $[7,21,25,28,30]$, Türkiye'deki çalışmalar sınırlı sayıdadır $[16,17,19]$. Yapılan bu çalışmalarda nekropsi sonrasında, Altın çakalda $\mathrm{Me}$ socestoides lineatus, Taenia polyacantha, Rictularia cahirensis [19], Uncinaria stenocephala [17] ve Joyeuxiella echinorhynchoides, Toxascaris leonina, Toxocara canis, Uncinaria stenocephala kaydedilmiştir [16]. İnsan sağlığını da etkileyen çok sayıda helmintin son konağ1 olduğu bilinmesine rağmen, örnek bulmadaki zorluklar nedeniyle özellikle Türkiye'de bu hayvanların helmint yükü ile ilgili çok az bilgi bulunmaktadır. Bu yüzden çakal gibi yabani karnivorların helmintlerinin belirlenmesine yönelik yapılan çalışmalarda elde edilen verilerin tamamının değerli olduğu düşünülmektedir.

$\mathrm{Bu}$ çalışmanın amacı, Türkiye'nin iki farklı bölgesinden (Çanakkale-Ayvacık ve Bolu-Kıbrıscık) elde edilen iki altın çakaldaki helmintlerin durumunu saptamak, bulunan helmintlerin zoonotik özellikleri ve Türkiye'ye komşu ülkelerdeki (Yunanistan, Bulgaristan, İran) altın çakallarda kaydedilen helmintlerin karşılaştırılması yapılmaktadır.

\section{Materyal ve Metot}

\section{Örneklerin Toplanması ve Saklanması}

Veteriner Kontrol Merkez Araştırma Enstitüsü Müdürlüğüne 2014 yılında Bolu-Kıbrıscık ve 2016 y1lında Çanakkale-Ayvacık'tan iki ölü erkek altın çakal gönderilmiş, nekropsileri Munson ve ark. [20]'nın belirttiği yönteme göre yapılmıştır. Kalp, karaciğer ve akciğer örnekleri helmintolojik inceleme yapılana kadar $-20^{\circ} \mathrm{C}$ 'de, ince ve kalın bağırsaklar ise önce olas1 Echinococcus sp. yumurtaların inaktif hale getirebilmek amaciyla en az bir hafta süreyle $-80^{\circ} \mathrm{C}$ 'de bekletilmiş, sonrasında inceleme yapılana kadar $-20^{\circ} \mathrm{C}$ 'de saklanmıştır [9]. Örnekler incelemeden bir gece önce $+4^{\circ} \mathrm{C}$ 'de çözdürülmüştür.

\section{Kalp, Akciğer ve Karaciğer Muayenesi}

Kalp örnekleri makas yardımıyla açılarak, atrium ve ventriküller makroskobik olarak Dirofilaria immitis ve Angiostrongylus vasorum yönünden incelenmiştir [24].

Karaciğer ve akciğer örneklerinin makroskobik incelenmesi sonunda, organlar steril makas yard1mıyla yaklaşık $2 \mathrm{~cm}^{3}$ 'lük parçalara ayrılarak, 11 k $\% 0,9^{\prime}$ luk fizyolojik tuzlu su (FTS) içerisinde $37^{\circ} \mathrm{C}$ etüvde 2-4 saat inkube edilmiştir. Süre sonunda örnekler musluk suyu altında $100 \mu$ m'lik elekten geçirilmiş, üste kalan miktar stereo mikroskopta $(\times 40)$ (Leica ${ }^{\circledR}$ M165 C) incelenmiştir [22].

\section{Sindirim Sistemi Muayenesi}

İnce ve kalın bağırsaklar Sedimentasyon ve Sayım Tekniği ile incelenmiştir [12]. Bağırsaklar beşer eşit parçaya bölünüp, tek ucu küt makas yardımıyla uzunlamasına açılmıştır. Bağırsak içeriği makroskobik olarak helmint yönünden kontrol edilmiştir. Ardından $100 \mu \mathrm{m}$ 'lik elekte yıkanmış, elek üstünde kalan içerik stereo mikroskop altında incelenmiştir. Bağırsak parçaları ise \%0,9'luk FTS bulunan şişelere alınarak, birkaç dakika şiddetli bir şekilde çalkalanmıştır. Süre sonunda bağırsak parçaları baş ve işaret parmağının arasına alınarak sıyrılmıştır. Kalan içeriğe ise sedimentasyon tekniği uygulanmıştır. Sediment porsiyonlara ayrilarak stereo mikroskop altında incelenmiştir. Bulunan helmintler teşhisleri yapılana kadar gliserin alkolde $+4^{\circ} \mathrm{C}$ 'de saklanmıştır $[12,18]$. Tespit edilen trematod ve cestod örnekleri Borax Carmen ile boyanırken [11], nematodlar laktofenol ile şeffaflandırılmıştır. Tespit edilen tüm helmintler tek tek incelenerek morfolojik özellikleri kaydedilmiş, tür teşhisleri ilgili literatürlere $[3,13$, 19, 29] göre yapılmıştır.

\section{Bulgular}

Çakallara ait kalp, karaciğer ve akciğer örneklerinde herhangi bir helmint ya da gelişim dönemine rastlanmazken, bağırsaklarda bulunan helmintler ve sayıları Tablo 1'de gösterilmiştir. Çanakkale'den gönderilen altın çakal bir helmint türü (Joyeuxiella echinorhyncoides) ile enfekte iken, Bolu'dan gönderilen çakalda beş farklı helmint türü (Alaria sp., Mesocestoides sp., Uncinaria stenocephala, Rictularia cahirensis ve Linguatula serrata nimfi) saptanmıştır (Şekil 1).

Karnivorlarda bulunması muhtemel Echinococcus sp. yumurtalarının inaktivasyonu amaciyla 
örnekler $-80^{\circ} \mathrm{C}$ 'de bekletilmiş, bu esnada çalıșmada tespit edilen helmintlerin, morfolojilerinin büyük ölçüde bozulduğu görülmüştür.

Yapılan ölçümlerde; ölçümü yapılan 6 dişi $U$. stenocephala örnekleri 7,72-10,43 mm uzunluğunda ve 290-340 $\mu \mathrm{m}$ genişliğinde bulunmuştur. Vulva, parazitin arka ucuna 2,25-3,55 $\mathrm{mm}$ mesafelerde olup, parazitin son 1/3'ünde bulunduğu kaydedilmiştir. Bu türde morfolojisinin bozulmadığı görülen bir erkek parazitten alınan ölçümlerde ise parazitin $6,714 \mathrm{~mm}$ boyunda ve $270 \mu \mathrm{m}$ eninde olduğu ölçülmüştür. Parazitin spikülümleri neredeyse birbirine eşit ve $650 \mu \mathrm{m}$ uzunluğunda olduğu ölçülmüsştür. Tespit edilen erkek Rictularia cahirensis ise 7,26 mm uzunluğunda ve $270 \mu \mathrm{m}$ eninde olduğu ölçülmüştür. Boylarının birbirine neredeyse eşit olduğu görülen spikülümlerin ise $217,5 \mu \mathrm{m}$ olduğu ölçülmüştür. Morfolojisi bozulmamış Linguatula serrata nimflerinin üçü ölçülmüş ve boyutlarının 6,06-8,20 $\mathrm{mm} \times 1236-1967 \mu \mathrm{m}$ olduğu ölçülürken, parazitin kütikulasında yaka şeklinde küçük dikenciklerin bulunduğu tespit edilmiștir. Ölçümü yapılan iki Alaria sp. örneğinin 2,53-2,62 $\mathrm{mm} \times 622-793 \mu \mathrm{m}$ boyutlarında olduğu görülmüştür. Mesocestoides sp. nin ise 10 gebe halkası ölçülmüş ve boyutlarının 1152-1397 × 1348-1912 $\mu \mathrm{m}$ olduğu ölçülmüştür. Joyeuxiella echinorhynchoides' in rostellumunda 19-22 sıra çengel bulunduğu tespit edilmiştir. Halka laterallerinde bulunan iki genital atrium halkaların ön 1/3'ünde yer almaktadır. Halkalarda bulunan cirrus keseleri ise 169-228 $\mu \mathrm{m}$ uzunluğunda saptanmiştır.

Tablo 1. Bağırsaklarda bulunan helmintler ve sayıları.

\begin{tabular}{lcc}
\hline Bulunan helmint türleri & Bolu & Çanakkale \\
\hline Alaria sp. & 2 & - \\
Joyeuxiella echinorhynchoides & - & 29 \\
Mesocestoides sp. & 11 & - \\
Uncinaria stenocephala & $10(7$ ○- 3 Љ) & - \\
Rictularia cahirensis & $2\left({ }^{\Uparrow}\right)$ & - \\
Linguatula serrata nimfi & 24 & - \\
\hline
\end{tabular}

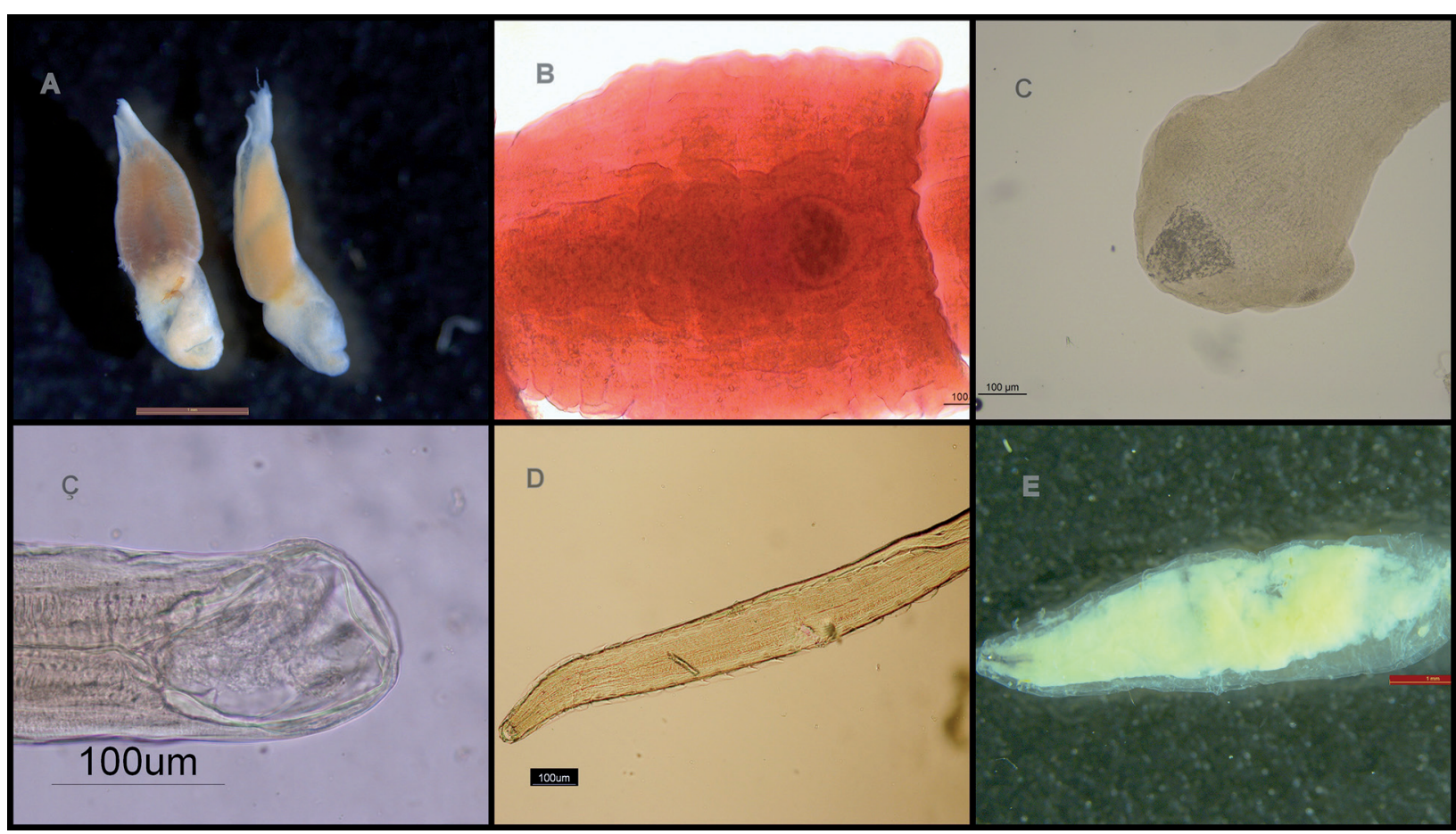

Şekil 1. A. Alaria sp. erişkin parazit, B. Mesocestoides sp. gebe halka, C. J. echinorhynchoides skoleksi Ç. U. stenocephala ön nihayeti D. $R$. cahirensis ön nihayeti ve tegümentteki dikenleri, E. L. serrata nimfi

\section{Tartışma ve Sonuç}

Altın çakallarda görülen; Alaria alata, Alaria canis, Heterophyes dispar, H. heterophyes, Trichinella sp.,
Toxocara sp., Ancylostoma sp., Joyeuxiella pasqualei, Dipylidium caninum, Mesocestoides lineatus, Macracanthomynchus sp. ve Echinococcus granulo- 
sus gibi diğer karnivorlara da bulaşabilen ve zoonoz olan helmintler daha önce çeşitli çalışmalarda bildirilmiştir [7, 10, 14, 15, 28]. Tespit edilen zoonoz helmint türleri, altın çakalların geniş yaşam alanlarına sahip olmaları ve günümüzde yaşam alanlar1nın şehirlere yaklaşması sebebiyle halk sağlığını da tehdit etmektedir. Ayrıca köpeklerin ve yabani karnivorların birbiriyle etkileşimi arttıkça enfeksiyonların da görülme ihtimali, yayılışı artmaktadır [14].

Türkiye'nin komşu ülkelerinde altın çakallarda yapılan helmint çalışmalarında saptanan türler birbirlerine benzerlik göstermektedir. Ancak araştırmalarda kullanılan örnek sayılarının Türkiye'de yapılan çalışmalara oranla fazla sayıda olması nedeniyle, bu ülkelerde saptanan helmint çeşitliliğinin de Türkiye'de saptananlara oranla daha fazla olmasının nedeni olduğu düşünülmektedir. Örneğin Yunanistan'da incelenen beş altın çakalda Alaria alata, Mesocestoides sp., Taenia pisiformis, Taenia sp., Toxocara canis, Uncinaria stenocephala, Ancylostoma caninum, Rictularia sp. türleri bulunmuştur [21]. İran'da bir çakalda Mesocestoides sp. ve kancalıkurt [30], 10 altın çakalda yapılan çalışmada Toxocara canis, Toxascaris leonina, Rictularia affinis, Taenia hydatigena, Dipylidium caninum, Mesocestoides lineatus, Joyeuxiella pasqualei, Dipylidium nolleri, Macroacanthorhynchus hirudinaceus, Macroacanthorhynchus sp., Alaria canis [7] ve 11 çakalda yapılan araştırmada ise Dipylidium caninum, Toxocara canis, Echinococcus granulosus, Toxascaris leonina, Diocthophyma renale, Taenia hydatigena, Ancylostoma caninum, Dirofilaria immitis [28] türleri bildirilmiştir. Sırbistan'da 447 altın çakalda 12 helmint türü saptanırken, en yaygin türlerin Mesocestoides lineatus, Alaria alata ve Toxocara canis olduğu kaydedilmiştir [6]. Ayrıca 60 altın çakalda yapılan başka bir çalışmada ise, 6 helmint türü teşhis edilmiş, bunlardan A. alata, Ancylostomatidae, T. canis en yaygın türler olarak kaydedilmiştir [14].

Çalışmamızda bulunan helmint türlerinden $R i$ ctularia cahirensis, Uncinaria stenocephala, Mesocestoides sp., ve Joyeuxiella echinorhynchoides daha önce Türkiye'de çakallarda bildirilmiştir [16, 17, 19]. Zoonotik önemi bulunan Alaria Türkiye'de daha önce tilkilerde [19] ve sokak köpeğinde [27] saptanmış, altın çakallarda ise kaydedilmemiştir. Ayrıca Linguatula serrata da daha sokak köpekle- rinden bildirilmiş $[1,8]$, ancak çakallarda herhangi bir bildirim yapılmamıştır. Bu çalışmada tespit edilen Alaria sp. ve L. serrata nimfi bildiğimiz kadarıyla Türkiye'de altın çakallardaki ilk bildirimlerdir.

Çakallarda tespit edilen ve zoonoz özelliği olan helmintlerin insanlara bulaşma yolları ve neden olduğu klinik tablolar incelendiğinde; insanlar enfeksiyona Alaria sp.'nin mezoserkerli paratenik konaklarının (kurbağa gibi) çiğ ya da az pişmiş olarak alınması ile yakalanmaktadır. Alınan mezoserkerlerin insanlarda direkt veya dolaşım yoluyla göz dahil tüm dokulara yayılabildiği ve insanlarda ölümlere neden olabildiği bildirilmiştir [27]. Benzer şekilde, Mesocestoides sp. tetrathyridiumları ile enfekte y1lan, tavuk ve av kuşları gibi ara konakların çiğ ya da az pişmiş olarak insanlar tarafindan tüketilmesi ile pleural ve peritoneal tetrathyridiosis oluşmakta, ciddi klinik belirtilere, hatta ölümlere neden olabileceği bildirilmektedir [23]. Uncinaria stenocephala L3'lerinin insan derisi ile temas etmesi durumunda, larvalar deriyi delerek Cutaneus Larva Migrans (Deri Larva Göçü) oluşturmakta, bu durum insanlarda dermatitislere neden olmaktadir [5]. Linguatula serrata'nın larval dönemleri de insanlarda görülen visceral pentastomiasis'in nedenlerinden biridir. Genelde asemptomatik görülse de, hepatik ya da pulmoner malignite şeklinde klinik ve radyolojik olarak görülebilmektedir [26]. Çalışmada tespit edilen helmintlerin tamamının, ya ara konakları ya da paratenik konakları çakalların yoğun olarak avladığ1 fare, rat, tavşan, kertenkele gibi sürüngenler ve kuşlardır [2]. Bu durum çakal gibi yabani karnivorların beslenme davranışları ile parazit çeşitliliği ve parazit yükleri arasında bağlantı olduğunu da göstermektedir. Ayrıca çalışmada tespit edilen tüm helmint türlerine köpeklerin de son konak olduğu bilinmektedir. Bu nedenle köpek kaynaklı helmint enfeksiyonlardan korunma ve eradikasyon ile ilgili yapılacak her türlü programda yabani karnivorların da göz önüne alınması, oluşturulacak programların başarı ihtimalini daha da arttıracağı düşünülmektedir. $\mathrm{Bu}$ nedenle oldukça geniş yayıllışa sahip bu karnivor türündeki parazitlerin belirlenmesi amacıyla daha geniş çaplı çalışmalar yapılarak bu hayvanların taşıdığ sağlığı, hem de hayvan sağlığının korunması için oldukça gereklidir. Bu bağlamda çalışmamızda elde edilen sonuçlar altın çakallardaki parazitler hakkında güncel veri niteliği taşımaktadır. 


\section{Kaynaklar}

1. Aldemir OS, (2004). Erzurum yöresi sokak köpeklerinde Linguatula serrata'nın yayılışı. Türkiye Parazitol Derg. 28 (1): 42-44.

2. Ambarlı H, Ertürk A, Soyumert A, (2016). Current status, distribution and conversation of Brown bear (Ursidae) and wild canids (gray wolf, golden jackals, and red fox; Canidae) in Turkey. Turk J Zool. 40, 944-956.

3. Anderson RC, (2000). Nematode parasites of vertebrates: their development and transmission. $2^{\text {nd }}$ ed.

4. Arnold J, Humer A, Heltai M, Murariu D, Spassov N, Hacklander K, (2012). Current status and distribution of golden jackals Canis aureus in Europe. Mammal Review. 42(1), 1-11.

5. Centers for Diseases Control and Prevention (2017). Hookworm. Erişim adresi: https://www.cdc.gov/dpdx/ hookworm/index.html, Erișim tarihi: 10.04.2019.

6. Cirovic D, Pavlovic I, Penezic A, Kulisic Z, Selakovic S, (2015). Levels of infection of intestinal helminth species in the golden jackals Canis aureus from Serbia. J Helminthol. 89, 28-33.

7. Dalimi A, Sattari A, Motamedi GH, (2006). A study on intestinal helminthes of dogs, foxes and jackals in the western part of Iran. Vet Parasitol. 142, 129-133.

8. Doğanay A, (1983). Ankara sokak köpeklerinde Linguatula serrata Frohlich, 1789 'un yayılışı ve halk sağlı̆̆ önemi. Ankara Univ Vet Fak Derg. 30 (4), 652-656.

9. Eckert J, Gottstein B, Heath D, Liu FJ, (2001). Prevention of echinococcosis in human and safety precautions. In: WHO/ OIE Manual on Echinococcosis in Humans and Animals: A Public Health Problem of Global Concern, edited by J. Eckert, M.A. Gemmell, F.X. Meslin, Z.S. Pawlowski. World Organisation for Animal Health, Paris, France, pp.: 238-246.

10. Gherman CM, Mihalka AD, (2017). A synoptic overview of golden jackal parasites reveals high diversity of species. Parasite Vector, 10, 419-459.

11. Gökçen, A, (2008). Helmintlerde Tespit, Boyama ve Kalıcı Preparat Yapımı. Türkiye Parazitoloji Dergisi, 32 (2): 177 - 181, 2008

12. Hofer S, Gloor S, Müller U, Mathis A, Hegglin D, Deplazes P, (2000). High prevalence of Echinococcus multilocularis in urban red foxes (Vulpes vulpes) and voles (Arvicola terrestris) in the city of Zürich, Switzerland. Parasitology. 120(2), 135-142.

13. Hrčkova G, Miterpakova M, O'connor A, Šnábel V, Olson PD, (2011). Molecular and morphological circumscription of Mesocestoides tapeworms from red foxes (Vulpes vulpes) in central Europe. Parasitology, 138 (5), 638-647.

14. Ilic T, Becskei Z, Petrovic T, Polacek V, Ristic B, Milic S, Stepanovic P, Radisavljevic K, Dimitrijevic S, (2016). Endoparasitic fauna of red foxes (Vulpes vulpes) and golden jackals (Canis aureus) in Serbia. Acta Parasitol. 61(2), 389-396.
15. Lahmar S, Boufana B, Ben Boubaker S, Landolsi F (2014). Intestinal helminths of golden jackals and red foxes from Tunisia. Vet Parasitol. 204(3-4), 297-303.

16. Merdivenci A, (1983). Son 30 yll (1952-1982) içinde Türkiye'de varlı̆̆ını ilk kez bildirdiğimiz parazitler. Türk Mikrobiol Cem Derg. 13, 23-38.

17. Merdivenci A, Buyurman Ü, (1965). The case of uncirariasis in a jakal in Turkey. Türk J Biol. 15(1-2), 52-60.

18. Meshgi B, Eslami A, Bahonar Ar, Kharrazian-Moghadam M, Gerami-Sadeghian A, (2009). Prevalence of parasitic infections in the red fox (Vulpes vulpes) and golden jackal (Canis aureus) in Iran. Iran J Vet Res. 10(4), 387-391.

19. Mimioğlu MM, Güralp N, Tolgay N, Sayın F, (1965). Ankara civarında tilki (Vulpes vulpes)lerde bulduğumuz helmintler. Ankara Üniv Vet Fak Derg. 12(3), 164-190.

20. Munson L, Karesh WB, Mcentee MF, Lowenstine LJ, Roelke-Parker ME, Williams E, Woodford MH, Haines D, (2006). Necropsy of wild animals. Wildlife Conservation Society. Erişim adresi: http://www.cldavis.org/ghpn/tools/ Necropsy\%20of\%20Wild\%20Animals.pdf, Erișim tarihi 17.04.2019.

21. Papadopoulos H, Himonas C, Papazahariadou M, Antoniadou-Sotiriadou K, (1997). Helminths of foxes and other wild carnivores from rural areas in Greece. $\mathrm{J}$ Helminthol. 71, 227-231.

22. Saeed I, Maddox-Hyttel C, Monrad J, Kapel CM, (2006). Helminths of red foxes (Vulpes vulpes) in Denmark. Vet Parasitol. 139(1), 168-179.

23. Széll Z, Tolnai Z, Sréter T (2015) Environmental determinants of the spatial distribution of Mesocestoides spp. and sensitivity of flotation method for the diagnosis of mesocestoidosis. Veterinary parasitology, 212(3-4), 427-430.

24. Şenlik, B. (2011). "Teşhis Yöntemleri”, Editör: Tınar R. Veteriner Helmintoloji. Bursa, Dora Basım Yayın.

25. Takacs A, Szabo L, Juhasz L, Takacs AA, Lanszki J, Takacs PT, Heltai M, (2014). Data on the parasitological status of golden jackal (Canis aureus L, 1758) in Hungary. Acta Vet Hung. 62(1), 33-41.

26. Tappe D, Büttner DW, (2009). Diagnosis of Human Visceral Pentastomiasis. PLoS Negl Trop Dis. 3(2), e320.

27. Umur Ş, (1998). Bir köpekte Alaria alata olgusu. Turk J Vet Anim Sci. 22, 89-92.

28. Vafae Eslahi A, Kia EB, Mobedi I, Sharifdini M, Badri M, Mowlavi G, (2017). Road killed carnivores illustrate the status of zoonotic helminthes in caspian sea littoral of Iran. Iran J Parasitol. 12(2), 230-235.

29. Yamaguti S (1961). Systema Helminthum Volume III The Nematodes of Vertebrates Part I. Interscience Publishers Inc., Newyork. 578-579.

30. Zare-Bidaki M, Mobedi I, Sadeghieh Ahari S, Habibizadeh S, Naddaf SR, Siavashi MR, (2010). Prevalence of zoonotic intestinal helminths of canids in moghan Plain, Northwestern Iran. Iran J Parasitol. 5(2), 42-51. 